\title{
SeaDataNet - Pan-European infrastructure for marine and ocean data management: unified access to distributed data sets
}

\section{Dick M.A. Schaap \& Roy K. Lowry}

To cite this article: Dick M.A. Schaap \& Roy K. Lowry (2010) SeaDataNet - Pan-European infrastructure for marine and ocean data management: unified access to distributed data sets, International Journal of Digital Earth, 3:S1, 50-69, DOI: 10.1080/17538941003660974

To link to this article: https://doi.org/10.1080/17538941003660974

曲 Published online: 14 Apr 2010.

Submit your article to this journal $๘$

山ll Article views: 167

Q View related articles ¿

47 Citing articles: 4 View citing articles 지 


\title{
SeaDataNet - Pan-European infrastructure for marine and ocean data management: unified access to distributed data sets
}

\author{
Dick M.A. Schaap ${ }^{a *}$ and Roy K. Lowry ${ }^{b}$ \\ ${ }^{a}$ MARIS BV, Voorburg, The Netherlands; ${ }^{b} B O D C$, Liverpool, UK \\ (Received 21 July 2009; final version received 14 January 2010)
}

\begin{abstract}
Data availability is of vital importance for marine and oceanographic research but most of the European data are fragmented, not always validated and not easily accessible. In the countries bordering the European seas, more than 1000 scientific laboratories from governmental organisations and private industry collect data using various sensors on board of research vessels, submarines, fixed and drifting platforms, aeroplanes and satellites to measure physical, geophysical, geological, biological and chemical parameters, biological species and others. SeaDataNet is an Integrated Research Infrastructure Initiative (I3) (2006-2011) in the EU FP6 framework programme. It is developing an efficient distributed Pan-European marine data management infrastructure for managing these large and diverse data sets. It is interconnecting the existing professional data centres of 35 countries, active in data collection and providing integrated databases of standardised quality on-line. This article describes the architecture and the features of the SeaDataNet infrastructure. In particular it describes the way interoperability is achieved between all the contributing data centres. Finally it highlights the on-going developments and challenges.
\end{abstract}

Keywords: ocean data; marine data; data management; infrastructure; interoperability

\section{Introduction}

Multidisciplinary oceanographic and marine data are collected by more than a thousand research institutes, governmental organisations and private companies in the countries bordering the European seas using various heterogeneous observing sensors installed on research vessels, submarines, aircraft, moorings, drifting buoys and satellites. The sensors measure physical parameters (temperature, salinity current, sea-level, optical properties, magnetic field and gravity), chemistry, biology, seabed characteristics, seabed depth, etc. The data are collected at a very considerable cost and are of prime value because they are the reference for any study and, if lost, cannot be remade.

This data and information is very important for research, but also for monitoring, predicting and managing the marine environment, assessing fish stocks and biodiversity, offshore engineering, controlling any hazard or disaster and the tourist industry. They support the execution of international protocols, conventions and agreements, which have been signed by coastal states for protection of the seas,

\footnotetext{
*Corresponding author. Email: dick@maris.nl 
such as Oslo Paris Commission (OSPAR), Helsinki Commission (HELCOM) and the Bucharest and Barcelona conventions. They are essential for implementation of Europe's environmental policy concerning Integrated Coastal Zone Management (ICZM), the Water Framework Directive, and the new Marine Strategy Directive. Overall there are many thousands of users, based in the research sector, government and industry.

SeaDataNet is an Integrated Research Infrastructure Initiative (I3) in EU FP6 to provide the Pan-European data management system adapted both to the fragmented observation system and the users need for integrated access to data, metadata, products and services. The SeaDataNet project started in 2006. It builds upon earlier data management infrastructure projects, undertaken over a period of 20 years by an expanding network of oceanographic data centres from the countries around all European seas. Its predecessor project Sea-Search had a strict focus on metadata. SeaDataNet maintains significant interest in the further development of the metadata infrastructure, but its primary objective is the provision of easy data access and generic data products.

The SeaDataNet partnership consists of well-established National Oceanographic Data Centres (NODCs), within the framework of the programme 'International Oceanographic Data and Information Exchange' of the 'Intergovernmental Oceanographic Commission' (IOC-IODE), oceanographic data focal points, major satellite data centres, expert modelling centres, experts in IT and three international bodies (International Council for the Exploration of the Sea (ICES), Intergovernmental Oceanographic Commission (IOC), Joint Research Centre (JRC)).

\section{Objectives}

The SeaDataNet project has the following objectives:

- To set-up and operate an efficient Pan-European distributed infrastructure for managing marine and ocean data by connecting 40 NODC's, national oceanographic focal points and ocean satellite data centres, in Europe. These data centres are mostly divisions of major national marine research institutes and based in 35 countries, surrounding the European seas.

- To ensure consistent dataset quality and to provide on-line trans-national access to marine metadata, data, products and services through a single portal, while the base data and information are stored and managed at the distributed data centres.

- To secure the long-term archiving of the large number of multidisciplinary data.

- To develop added value, regional data products like gridded climatologies and trends, in partnership with scientific research laboratories.

\section{Planning}

The SeaDataNet project is delivering and operating the infrastructure in three versions:

- Version 0: maintenance and further development of the metadata systems developed by the pre-decessor Sea-Search project. 
- Version 1: harmonisation and upgrading of the metadatabases through adoption of the ISO 19115 metadata standard and provision of trans-parent data access and download services from all partner data centres through upgrading the Common Data Index (CDI) services and deployment of a data object delivery service.

- Version 2: adding data product services and OGC compliant viewing services and further virtualisation of data access.

\section{SeaDataNet Version 0}

The SeaDataNet portal has been set-up at http://www.seadatanet.org and it provides a platform for all SeaDataNet services and standards as well as background information about the project and its partners. The Sea-Search project focused on metadata and designed and populated an array of Pan-European discovery services of marine data and information resources. These services are available for public users with dedicated on-line user interfaces and are maintained by SeaDataNet partners with entries from all the 35 countries.

\section{European Directory of Marine Environmental Data (EDMED)}

The European Directory of Marine Environmental Data (EDMED) was initiated in 1991 by the British Oceanographic Data Centre (BODC). It was updated and expanded during the European Network for Oceanographic Data \& Information Management (EURONODIM) and Sea-Search projects and has recently been developed still further by SeaDataNet. It is a high-level inventory of datasets relating to the marine environment. EDMED is a comprehensive reference to the marine data and sample collections held within Europe providing marine scientists, engineers and policy-makers with a simple discovery mechanism. It covers all marine environmental disciplines. At present, EDMED describes more than 3300 data sets, held at over 630 Data-Holding Centres from countries across Europe.

\section{Cruise Summary Report (CSR)}

The report of observations/samples collected by oceanographic programmes (ROSCOP) was conceived by IOC/IODE in the late 1960s in order to provide a coarse-grained inventory for tracking oceanographic data collected by research vessels. The ROSCOP form was extensively revised in 1990, and was re-named the Cruise Summary Report (CSR). Most marine disciplines are represented in the CSR, including physical, chemical and biological oceanography, fisheries, marine contamination/pollution, and marine geology and meteorology.

Traditionally, it is the Chief Scientist's obligation to submit a CSR to his/her NODC not later than two weeks after the cruise. These have been periodically transmitted to the World Data Centres for Oceanography and to ICES.

In the late 1980s ICES led the effort to digitise the ROSCOP/CSR information and pioneered the development of a database for this information, and, in collaboration with IOC/IODE, developed and maintained a PC-based CSR entry tool and search facility. The emphasis for this was on ICES member countries, but extended to other countries who wished to submit information. The CSR activity 
gained new momentum in Europe during the EURONODIM/Sea-Search projects and is being further developed in SeaDataNet under the lead of BSH/DOD, Germany. The combined ICES and Sea-Search/SeaDataNet CSR database now comprises details of over 35,000 oceanographic research cruises primarily from Europe and North America, but also including some other regions (e.g. Japan and Australia), with some information extending as far back as the 1940s.

The CSR metadatabase contains details of completed cruises and provides summary information of oceanographic measurements made and samples taken.

\section{European Directory of Marine Environmental Projects (EDMERP)}

The European Directory of Marine Environmental Projects (EDMERP) was initiated in the EURONODIM project and it gives an overview of research projects relating to the marine environment. It covers all marine environmental disciplines. Research projects are catalogued as factsheets with their most relevant aspects. The primary objective is to support users in identifying interesting research activities and in connecting them to involved research managers and project results like data, models, publications, etc. across Europe. Currently, EDMERP describes more than 1800 Research Projects, from a wide range of disciplines, from over 300 research institutes.

\section{European Directory of the Ocean-Observing System (EDIOS)}

The European Directory of the Ocean-Observing System (EDIOS), an initiative of EuroGOOS, gives an overview of the ocean measuring and monitoring systems operated by European countries. The directory is a prerequisite for the full implementation of EuroGOOS providing an inventory of the continuously available data for operational models. This information provides the basis for optimal deployment of new instruments, and the design of sampling strategies. This directory includes discovery information on location, measured parameters, data availability, responsible institutes and links to data-holding agencies plus some more technical information on instruments such as sampling frequency. The EDIOS directory currently holds well over 12,000 data entries.

\section{European Directory of Marine Organisations (EDMO)}

The European Directory of Marine Organisations (EDMO) contains the contact information and activity profiles for the organisations whose data are described by the metadatabases. In the early days this was poorly co-ordinated with information of this type held in each metadatabase. However, during SeaDataNet a central organisations database has been set-up, maintained by project partners through a Content Management System (CMS), which streamlines the information management and ensures that information is internally consistent. EDMO at present covers more than 1500 addresses and profiles of organisations.

\section{Common Data Index (CDI)}

The CDI gives detailed insight into the available data objects in partners' databases paving the way to SeaDataNet's ultimate objective of direct on-line data access. The 
CDI was initiated as a pilot within Sea-Search. For purposes of standardisation and international exchange the ISO 19115 metadata standard was adopted and the CDI format defined by profiling this standard. Within SeaDataNet V0 the Sea-Search CDI version has been populated with entries from all SeaDataNet data centres. Currently, the CDI V0 metadatabase contains more than 340,000 CDI entries from 36 data centres in 29 countries across Europe, covering many data types.

The CDI database is accessed through an on-line interface that gives users detailed insight on the availability and geographical distribution of the marine data archived at the connected data centres. It also provides sufficient information to allow the user to assess data relevance. Moreover it gives links for on-line access to data available from partner websites or for on-line request services. The CDI V0 gave users a unified index, but users still had to negotiate a multitude of different data access interfaces (see Figure 1).

\section{Approach for SeaDataNet V1 and V2}

The approach for SeaDataNet V1 and V2 comprises developing the following services:

- $\quad$ Discovery services $=$ Metadata directories.

- $\quad$ Security services $=$ Authentication, Authorisation and Accounting (AAA).

- $\quad$ Delivery services $=$ Data access and downloading of datasets.

- $\quad$ Viewing services $=$ Visualisation of metadata, data and data products.

- $\quad$ Product services $=$ Generic and standard products.

- Monitoring services $=$ Statistics on usage and performance of the system.

- $\quad$ Maintenance services $=$ Updating of metadata by SeaDataNet partners.

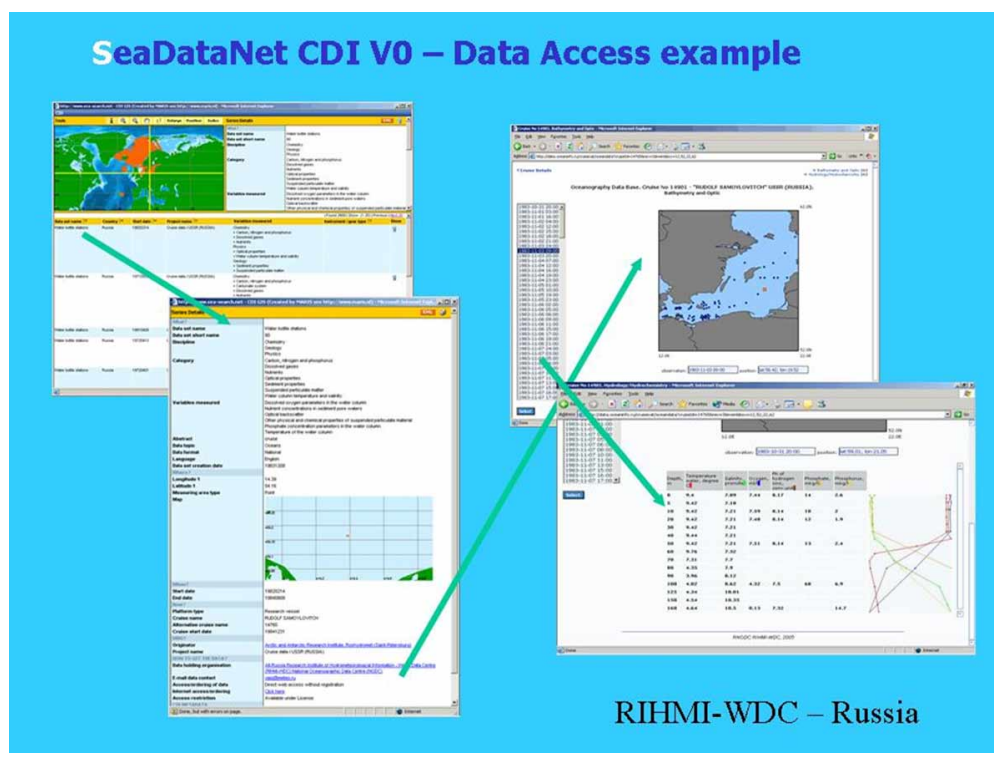

Figure 1. CDI V0 data search and retrieval dialogue - for example, Russian data centre. 
These services are operated over a distributed network of interconnected data centres accessed through a central portal (http://www.seadatanet.org). In addition to service access, the portal provides information on data management standards, tools and protocols.

The architecture has been designed to provide a coherent system based on V1 services, whilst leaving the pathway open for later extension with V2 services. For the implementation, a range of technical components have been defined. The majority are operational with the remainder in the final stages of development and testing. These make use of recent web technologies, and also include Java components, to provide multi-platform support and syntactic interoperability. To facilitate sharing of resources and interoperability, SeaDataNet has adopted SOAP Web Service technology. Software applications written in various programming languages on a range of platforms can use web services to exchange data over the internet in a manner similar to inter-process communication on a single computer. The SeaDataNet architecture and components have been designed to handle all kinds of oceanographic and marine environmental data including both in-situ and remotely sensed observations.

\section{Interoperability}

Interoperability is the key to distributed data management system success and it is achieved in SeaDataNet by:

- Using common quality control protocols and flag scale.

- Using controlled vocabularies from a single source that have been developed using international content governance.

- Adopting the ISO 19115 metadata standard for all metadata directories.

- Providing XML validation services to quality control the metadata maintenance, including field content verification based on Schematron.

- Providing standard metadata entry tools.

- Using harmonised Data Transport Formats (NetCDF, Ocean Data View ODV ASCII and MedAtlas ASCII) for data set delivery.

- Adopting of OGC standards for mapping and viewing services.

- Using SOAP web services in the SeaDataNet architecture.

\section{Quality control guidelines}

A guideline (V1) of recommended quality control (QC) procedures has been compiled, reviewing NODC schemes and other known schemes (e.g. WGMDM guidelines, World Ocean Database, GTSPP, Argo, WOCE, QARTOD, ESEAS, SIMORC, etc.). The guideline at present contains QC methods for conductivity, temperature, depth (CTD) (temperature and salinity), current meter data (including accoustic Doppler current profiler (ADCP)), wave data and sea-level data. Furthermore a scheme of QC flags to be used in SeaDataNet to label individual data values has been defined and adopted.

The guideline (V1) has been compiled in discussion with IOC, ICES and Joint WMO-IOC Technical Commission for Oceanographic and Marine Meteorology (JCOMM), to ensure an international acceptance and tuning. Important feedback 
originated from the joint IODE/JCOMM Forum on Oceanographic Data Management and Exchange Standards (January 2008), joined by SeaDataNet and international experts to consider on-going work on standards and to seek harmonisation, where possible.

Activities are now underway for extending the guideline with QC methods for surface underway data, nutrients, geophysical data and biological data. The new guideline (V2) is expected around summer 2009.

\section{Common vocabularies}

Use of common vocabularies in all metadatabases and data formats is an important prerequisite for consistency and interoperability. Common vocabularies consist of lists of standardised terms that cover a broad spectrum of disciplines of relevance to the oceanographic and wider community. Using standardised sets of terms solves the problem of ambiguities associated with data markup and also enables records to be interpreted by computers. This opens up data sets to a whole world of possibilities for computer aided manipulation, distribution and long-term reuse. Therefore common vocabularies were set-up and populated by SeaDataNet.

New vocabulary technical governance is based on the NERC DataGrid (NDG) Vocabulary Server Web Service application programming interface (API). Nonprogrammatic access is provided to end-users by a client interface for searching, browsing and CSV-format export of selected entries. The API is compliant to WS Basis Profile 1.1, which is adopted as standard for all Web Services in SeaDataNet. The Vocabulary Server is populated with lists describing a wide range of entities relevant to marine metadata and data such as parameters, sea area names, platform classes, instrument types and so on.

Content governance of the vocabularies is very important and is done by a combined SeaDataNet and MarineXML Vocabulary Content Governance Group (SeaVoX), moderated by BODC, and including experts from SeaDataNet, MMI, MOTIIVE, JCOMMOPS and more international groups. SeaVox discussions are based on an email list server.

\section{Common data transport formats}

As part of the V1 services, data sets are accessible via download services. Delivery of data to users requires common data transport formats, which interact with other SeaDataNet standards (Vocabularies, Quality Flag Scale) and analysis and presentation tools (ODV and Data-Interpolating Variational Analysis - DIVA). Therefore the following formats have been defined:

- SeaDataNet ODV4 ASCII for profiles, time series and trajectories.

- SeaDataNet MedAtlas as optional extra format.

- NetCDF with CF compliance for gridded data sets.

The ODV4 and MedAtlas have been extended with a SeaDataNet semantic header. International cooperation is underway from SeaDataNet with the CF community and UNIDATA for a common NetCDF format (Common Data Model - CDM) for the oceanographic and meteorological domains, including a semantic header 


\section{Authentication, authorization and administration}

All the metadata systems and the website including the information on standards and tools are public domain. However, a single sign-on system is required for access to the distributed databases with data sets. This is not done to set-up a threshold for users, but to ensure that users agree with the SeaDataNet data policy and to allow SeaDataNet to become acquainted with its user community.

Users must register once in order to get a personal login name and password. This is done via a web form to provide the necessary information. In this process, the user is required to accept the terms and conditions of the 'SeaDataNet User Licence'. After processing, the user will receive his access credentials by email. The so-called Authentication, Authorization and Administration services (AAAS) are based upon a centralised conditional access system (CAS). SeaDataNet maintains and uses a central user register, but the allocation of the user roles determining data access rights, are managed at the national level by SeaDataNet partners.

The User Licence is part of the SeaDataNet Data Policy, that is intended to be fully compatible with the Directive of the European Parliament and of the Council on public access to environmental information, the INSPIRE Directive and the data access principles of IOC, ICES, WMO, GCOS, GEOSS and CLIVAR. The licence incorporates the following terms:

(1) The licensor grants to the licensee a non-exclusive and non-transferable licence to retrieve and use data sets and products from the SeaDataNet service in accordance with this licence.

(2) Retrieval, by electronic download and the use of Data Sets is free of charge, unless otherwise stipulated.

(3) Regardless of whether the data are quality controlled or not, SeaDataNet and the data source do not accept any liability for the correctness and/or appropriate interpretation of the data. Correct and appropriate data interpretation should follow scientific rules and is always the user's responsibility.

(4) Users must acknowledge data sources. It is not ethical to publish data without proper attribution or co-authorship. Any person making substantial use of data must communicate with the data source prior to publication, and should consider the data source(s) for co-authorship of published results.

(5) Data users should not give any SeaDataNet data or product to third parties, without prior consent from the source data centre.

(6) Data users must respect any and all restrictions on the use or reproduction of data. The use or reproduction of data for commercial purpose might require prior written permission from the data source.

\section{SeaDataNet V1 discovery services}

The V1 discovery services build upon the V0 directory services, but considerable work has been undertaken to upgrade and harmonise each of the directories wherever possible. The logical format of each directory has been reviewed and streamlined and an XML schema developed by ISO 19115 profiling. For common fields, common vocabularies have been defined or adopted, including additional population where necessary. Also the Vocabulary Server API has been upgraded for better support to the editing and retrieval tools. 
Maintenance workflow has been defined and developed for each of the directories, supporting the production and exchange of updates as XML files. Depending on the directory, the following maintenance mechanisms are provided (see Figure 2):

- On-line maintenance via on-line CMS.

- XML export from local system.

Local XML export can be produced by partners by:

- Using a data entry for interface provided by the MIKADO Java tool. MIKADO interacts with the web services of the vocabularies, EDMO and EDMERP and produces valid XML files that can be imported into the central V1 directories.

- Using MIKADO with a configuration file to export from local database(s) thereby generating XML files in bulk.

- Using partners' own software.

The XML files produced may be validated at the source prior to directory submission by an XML validation web service that both verifies document structure and checks field content against the Vocabulary Server.

Finally new user interfaces have been defined and implemented for each of the directories (see Figure 3).

\section{SeaDataNet V1 delivery services}

An important objective of the V1 system is to provide trans-parent access to the distributed data sets via a single user interface at the SeaDataNet portal and download service.

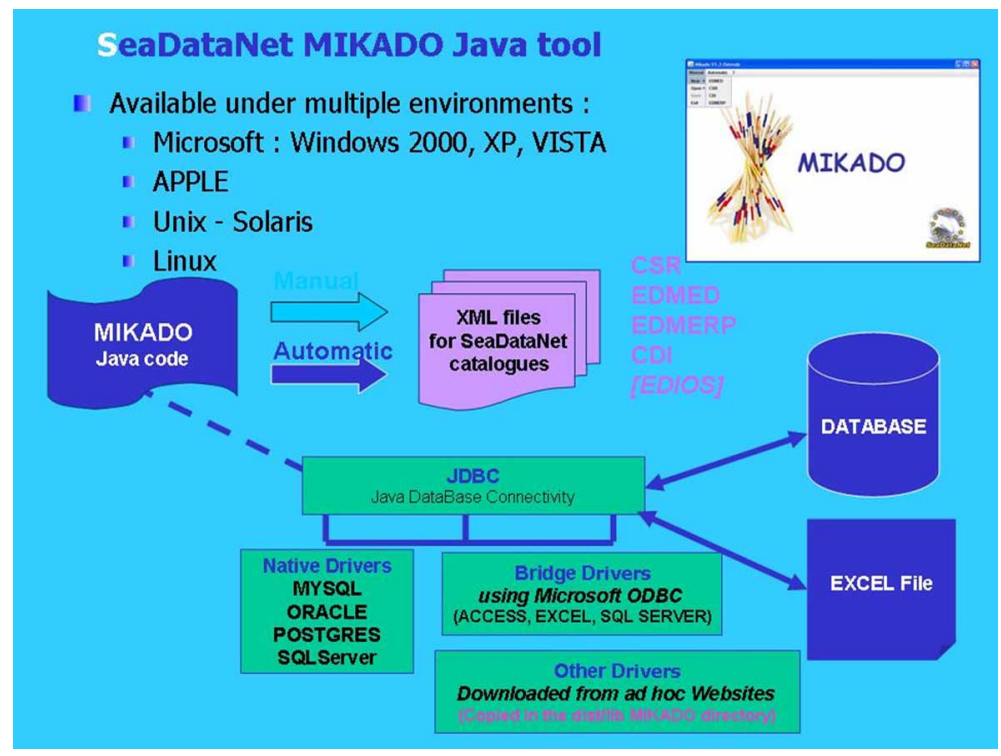

Figure 2. MIKADO editing tool. 


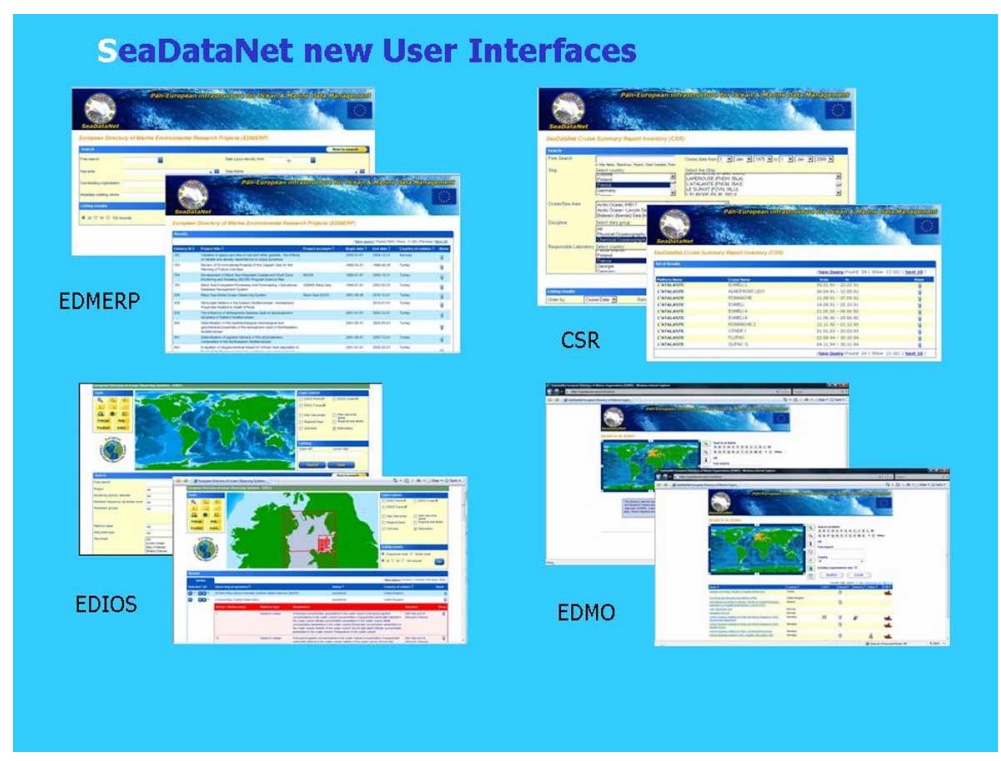

Figure 3. SeaDataNet V1 user interfaces.

In the SeaDataNet V1 architecture the CDI V1 provides the link between discovery and delivery. The CDI user interface enables users to have detailed insight of the availability and geographical distribution of marine data, archived at the connected data centres, and it provides the means for downloading data sets in common formats via a transaction mechanism.

The SeaDataNet portal provides registered users access to these distributed data sets via the CDI V1 Service (see Figure 4). There are two user interfaces:

- CDI V1 - Quick Search.

- CDI V1 - Extended Search.

Both interfaces enable users to search for data sets using a set of criteria. The selected data sets are listed. Geographical locations are indicated on a map. Clicking on the display icon retrieves the full metadata of the data set. This gives information on the what, where, when, how and who of the data set. It also gives standardised information on the data access restrictions that apply. The interfaces also feature a shopping mechanism, by which selected data sets can be included in a shopping basket (see Figure 5).

All users can freely query and browse in the CDI V1 directory; however, submitting requests for data access via the shopping basket requires that users are registered in the SeaDataNet central user register, thereby agreeing with the overall SeaDataNet User Licence.

All data requests are forwarded automatically from the SeaDataNet portal to the relevant data centres. This process is controlled via the Request Status Manager ( RSM) service at the portal, that communicates with the data centres via the Download Manager (DM) java software module, implemented at each of the data centres. Users receive a confirmation email of their data set requests and a link to the RSM service. By log-in to the RSM service users can check the status of their 


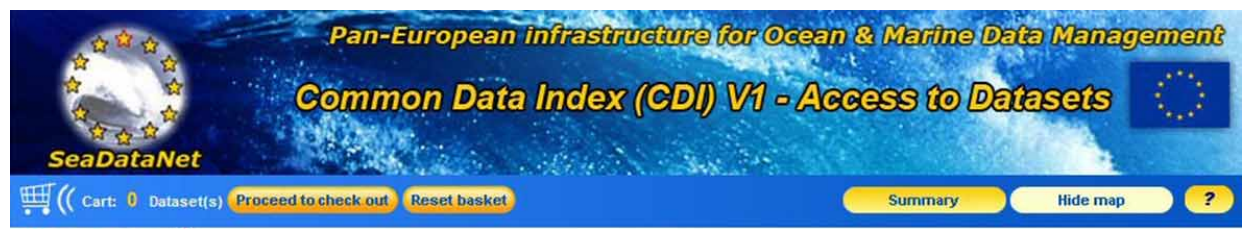

Reset all steps > point $邓$
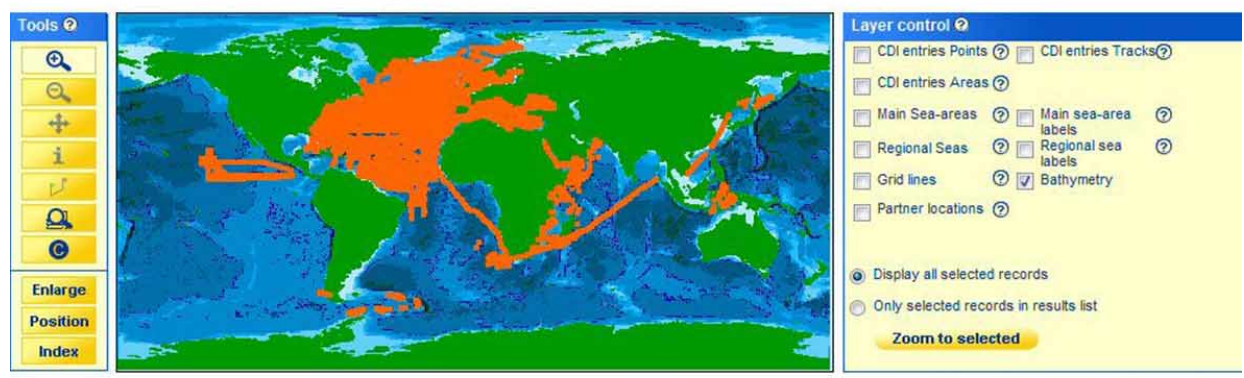

Search by:
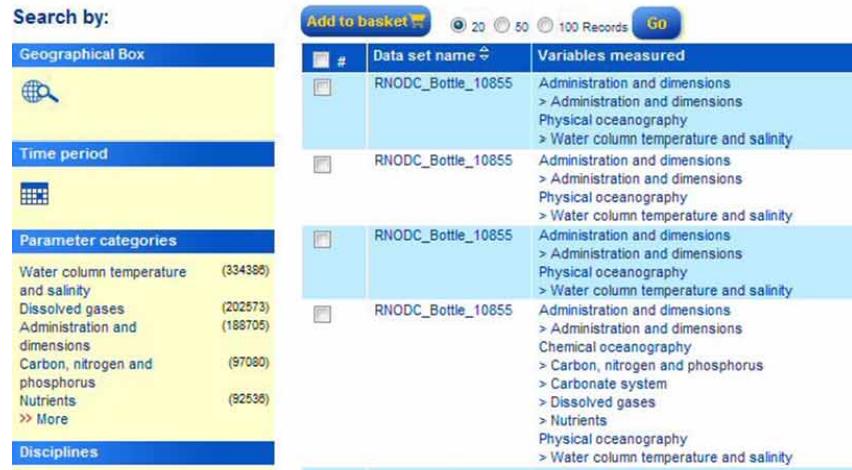

| Found 457958 | Show (1-20) | Previous | Hext 20

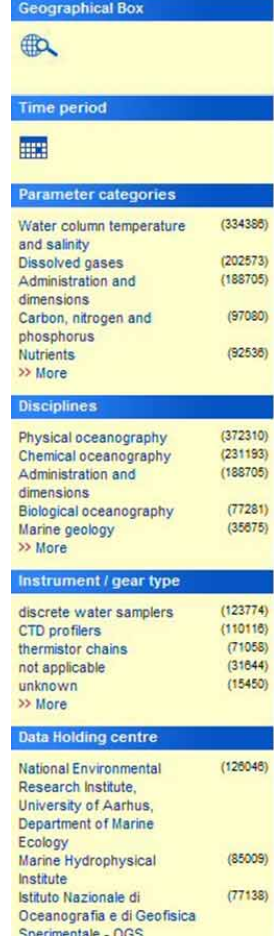
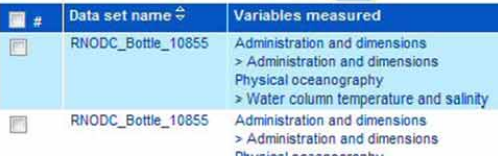

Administration and dimensions

$>$ Administration and dimensions

Physical oceanography
> Water column temperature and salinity

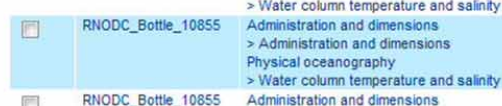

$>$ Administration and dimensions

Chemical oceanography

$>$ Carbon, nitrogen and phosphorus

$>$ Carbonate system

$>$ Dissolved gases

$>$ Nutrients

Physical oceanography

$>$ Water column temperature and salinty

Administration and dimensions

$>$ Administration and dimensions

$>$ Water column temperature and salinity

Administration and dimensions

Administration and dimensions

Physical oceanography

Administation lemperature and sainty

Administration and dimensions

> Administration and dimens
Physical oceanography

$>$ Water column temperature and salinity

\begin{tabular}{|l|l}
\hline Instrument $/$ gear type & $\Delta$
\end{tabular}

$\square$ RNODC_Botse

[1] RNODC_Bottle_10855

$\rightarrow$ Administration and dimensions

Physical oceanography

discrete water samplers 8

[1] RNODC_Bottie_10855

Water column temperature and salinity

\begin{tabular}{|c|c|c|c|c|}
\hline & & 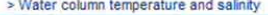 & & \\
\hline$\square$ & RNODC_Bottle_10855 & $\begin{array}{l}\text { Administration and dimensions } \\
\text { > Administration and dimensions } \\
\text { Physical oceanography } \\
\text { > Water column temperature and salinty }\end{array}$ & discrete water samplers & 3 \\
\hline 回 & RNODC_Bottle_1085S & $\begin{array}{l}\text { Administration and dimensions } \\
\text { > Administration and dimensions } \\
\text { Physical oceanography } \\
\text { > Water column temperature and salinity }\end{array}$ & discrete water samplers & 8 \\
\hline 回 & RNODC_Bottle_10855 & $\begin{array}{l}\text { Administration and dimensions } \\
\text { > Administration and dimensions } \\
\text { Physical oceanography }\end{array}$ & discrete water samplers & 3 \\
\hline
\end{tabular}

Figure 4. CDI V1 Quick Search interface.

requests and download data sets from the associated data centres, after access has been granted. Each CDI V1 metadata record includes a data access restriction tag. It indicates under which conditions the data set is accessible to users. Its values can vary from 'unrestricted' to 'no access' with a number of values in between. During registration every user will be qualified by its national NODC/Marine Data Centre 


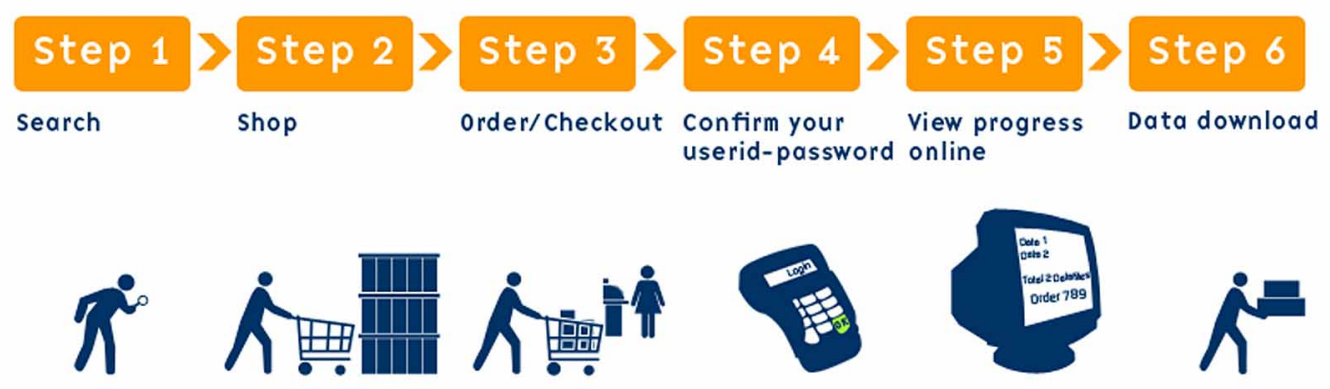

Figure 5. Process flow from search to delivery. 


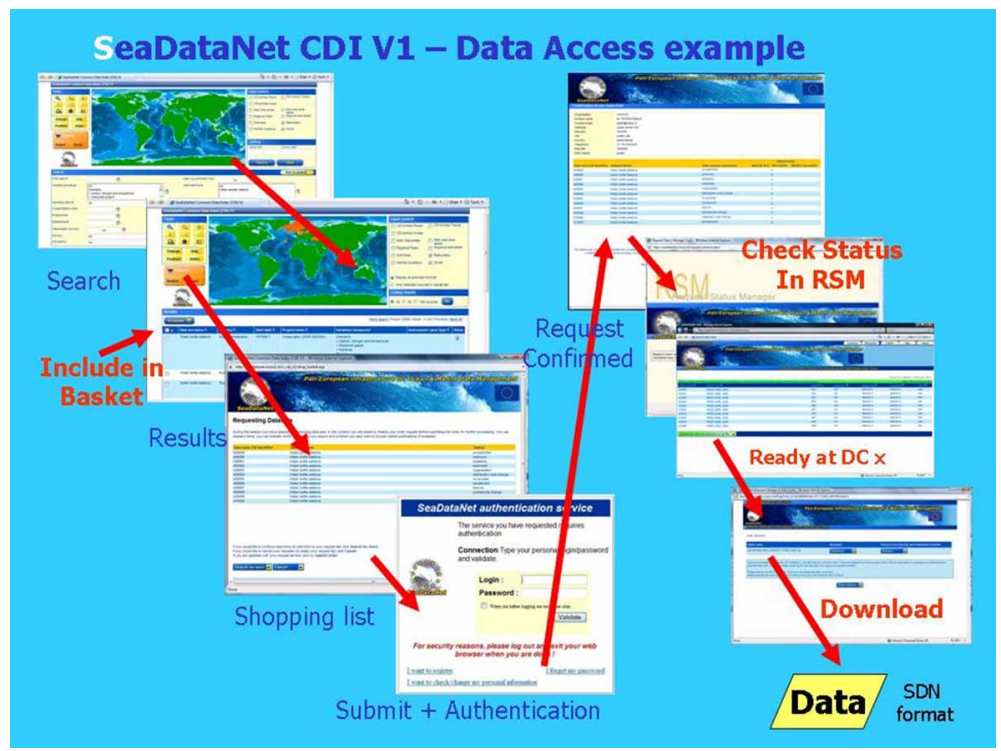

Figure 6. CDI V1 unified data search and retrieval dialogue.

with one or more SeaDataNet roles. The RSM service compares the given data access restriction for each data set requested, with the role(s) of the user as registered in the SeaDataNet central user register. This determines per data set request, whether a user gets direct access automatically, whether they first have to be considered by the data centre, that therefore might contact the user, or that no access is given. The actual delivery of data sets is done between the user and the selected data centre (see Figures 6 and 7).

\section{Presentation and analysis tools and data products}

SeaDataNet has adopted the freely available ODV software package as its fundamental data analysis and visualization software. ODV provides an interactive

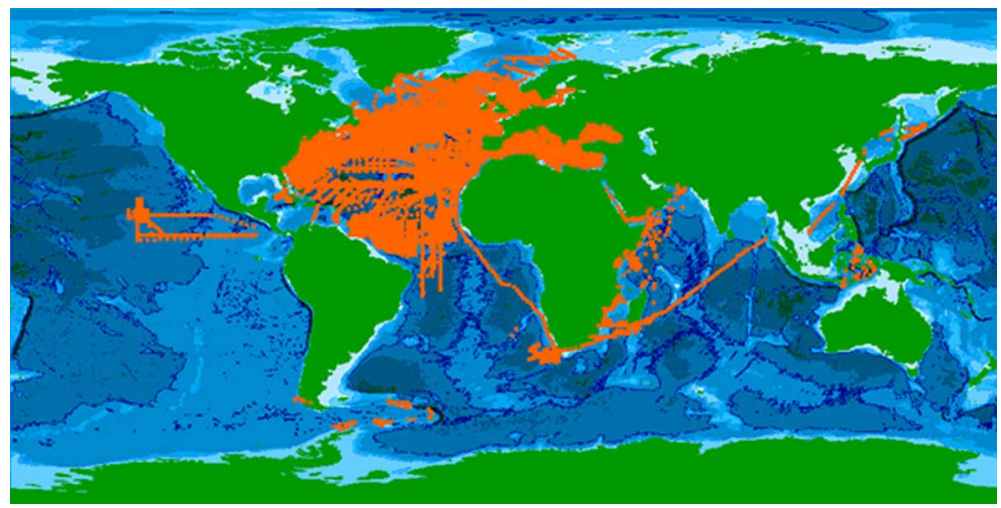

Figure 7. CDI V1 coverage at mid June 2009: ca. 450,000 CDI entries so far from 13 Data Centres; connecting other SeaDataNet partners is well underway. 
exploration, analysis and visualization of oceanographic and other geo-referenced profile or sequence data. It is available for all major computer platforms and currently has more than 10,000 registered users. ODV has a very rich set of interactive capabilities and supports a very wide range of plot types. This makes ODV ideal for visual and automated quality control. The latest release, ODV4, developed as part of SeaDataNet by partner AWI (Germany), overcomes many limitations of previous versions and now supports more flexible metadata models, an unlimited number of variables and custom quality flag schemes, and is fit for loading data sets in the SeaDataNet ODV ASCII format.

The ODV software is also being used in SeaDataNet for producing generic data products for various variables in each of the regional seas. In practice, in-situ measurements can be sparse and heterogeneously distributed. The DIVA software tool allows spatial interpolation (or analysis) of those observations on a regular grid in an optimal way. The analysis is performed on a finite element grid allowing for a spatially varying resolution and a good representation of the coastline and isobaths. The finite-element grid of DIVA will be able to adequately resolve even complex coastlines.

It is also possible to compute error maps for the gridded fields, which reflect the accuracy of the observations and their distribution. This allows assessment of the reliability of the gridded fields and to objectively identify areas with poor coverage. In an approach similar to generalised cross-validation, the value of the gridded fields can also be computed, without taking a particular observation into account. By comparing this analysed value with the observations, one can establish how consistent one particular observation is with the remaining dataset. This information can be used by data centres to identify bad data.

As a part of SeaDataNet, the DIVA method has been integrated into ODV, and the integration greatly facilitates the usage of DIVA. Features supported by the ODV/DIVA integration include proper treatment of domain separation due to land masses and undersea ridges or seamounts, and the realistic estimation of water mass properties on both sides of the divides. This is important in areas such as the Kattegat, with many islands separated by narrow channels.

The DIVA software can also load data sets prepared via the MEDATLAS QC software and other in-house QC systems. The command line interface of DIVA allows for batch processing of large data sets (see Figures 8 and 9).

The SeaDataNet website (http://www.seadatanet.org) has been completely overhauled and now gives overview, background information and access to the latest releases of the standards, tools and services of SeaDataNet.

\section{SeaDataNet further developments and challenges}

There are a number of challenges and further developments foreseen for SeaDataNet:

- To be compliant with the EU INSPIRE Directive, which is defining and delivering its Implementing Rules.

- To implement the V2 system configuration for generating SeaDataNet products for regional seas in Europe in an operational way. 


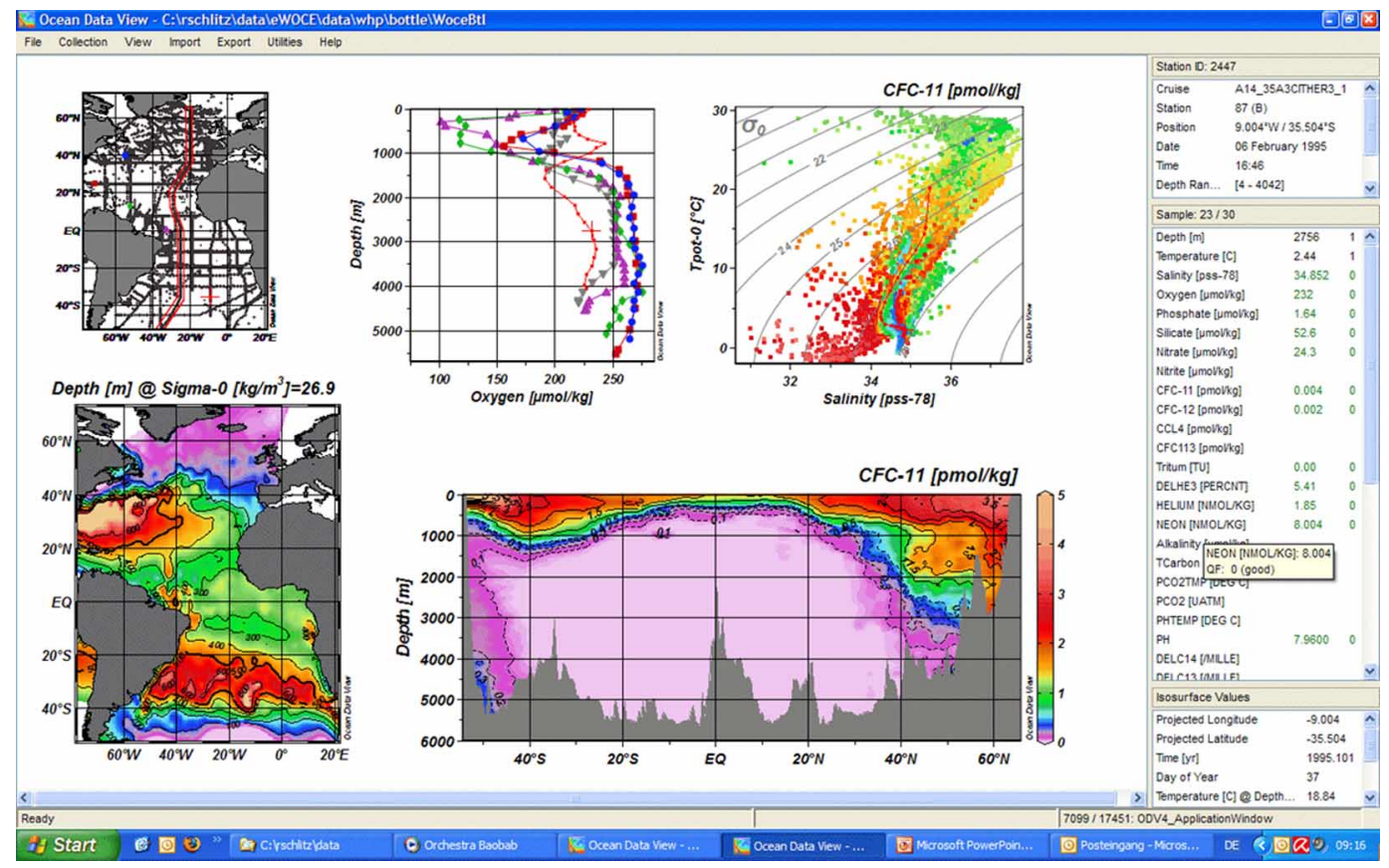

Figure 8. ODV analysis and presentation software. 

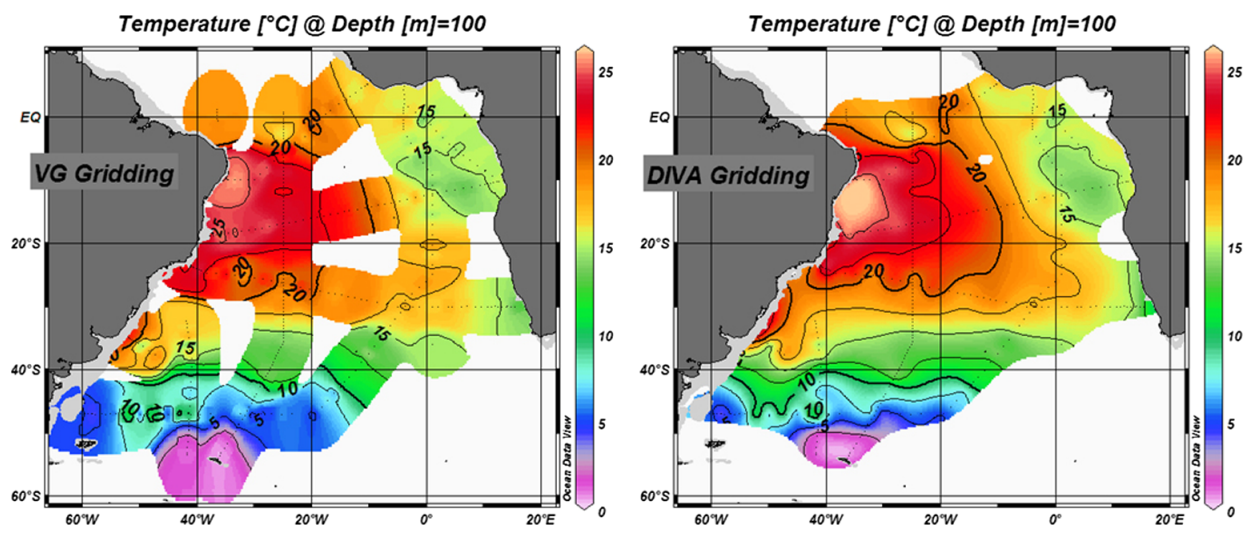

Figure 9. Example of improved smoothing using DIVA software.

- To expand the SeaDataNet data coverage in Europe to other marine and ocean disciplines and their actors, partly by extending the SeaDataNet infrastructure and partly by realising interoperability with other existing infrastructure systems.

- To contribute to the development of new standards and/or to adopt new standards, thereby securing that the infrastructure maintains operational.

- To secure interoperability with other infrastructures worldwide.

\section{Considering INSPIRE}

SeaDataNet started its work in 2006, basing its metadata schema upon the then available ISO 19115 DTD. Since then the ISO 19139 schema has become mature and is now part of the INSPIRE Implementing Rules as well as the Catalogue Service for the Web (CSW) ISO AP Version 2.0.2 (OGC). Therefore SeaDataNet is undertaking a pilot for implementing the CSW harvesting mechanism for metadata exchange between data centres and portal and exploring how to upgrade the SeaDataNet metadata XML files to ISO 19139. Full adoption will have an impact on all tools that SeaDataNet has developed so far for generating, editing, exchanging and importing metadata. The technical adoption and then full uptake by all partners will take a lot of effort and time. Therefore SeaDataNet is also working on a front-end solution, that will assure that the output from the SeaDataNet portal is INSPIRE compliant in the coming months. This is realised in cooperation with the University of Firenze (Italy) by applying their GI-CAT broker/mediation service. GI-CAT can harmonise metadata of various resources to ISO 19115 core profile and then produce CSW-ISO output (see Figures 10 and 11).

\section{Considering SeaDataNet product services}

These will provide generic regional products from available datasets and require delivery and viewing services. Preliminary products have been developed using existing data sets, and the ODV and DIVA analysis tools. The statistical output is formatted in NetCDF files that are made visible via Open Geospatial 


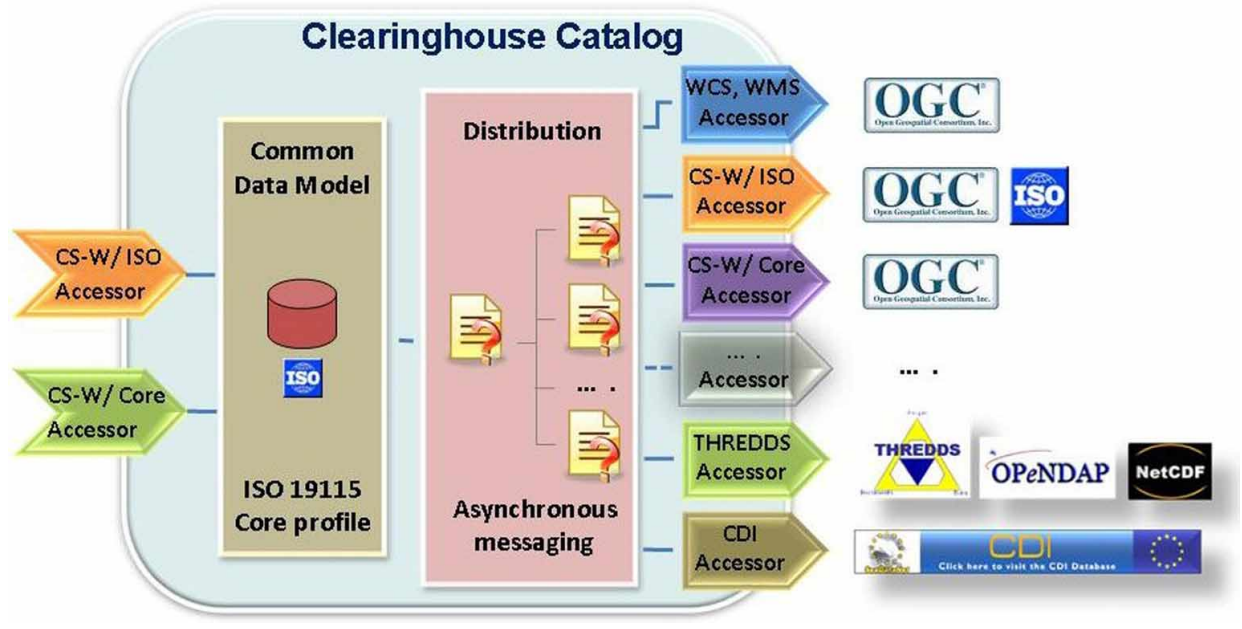

Figure 10. GI-CAT broker/mediation service.

Consortium-Web Map Services (OGC-WMS). Further work will be undertaken for expanding and improving these product viewing services (see Figure 12).

\section{Considering the expansion of SeaDataNet}

There are several activities underway. An important perspective is the recent adoption of the new Marine Directive by the EU, which includes a requirement for an overarching European Marine Observation and Data Network (EMODNET), that would improve availability of high quality data. The detailing of EMODNET is co-ordinated by an EU Taskforce, that has produced a draft roadmap. As part of the roadmap and analysis for the future EMODNET the EU launched a call for tenders for creating pilot components of EMODNET in July 2008. The overall objective is to migrate fragmented and inaccessible data into interoperable, continuous and publicly

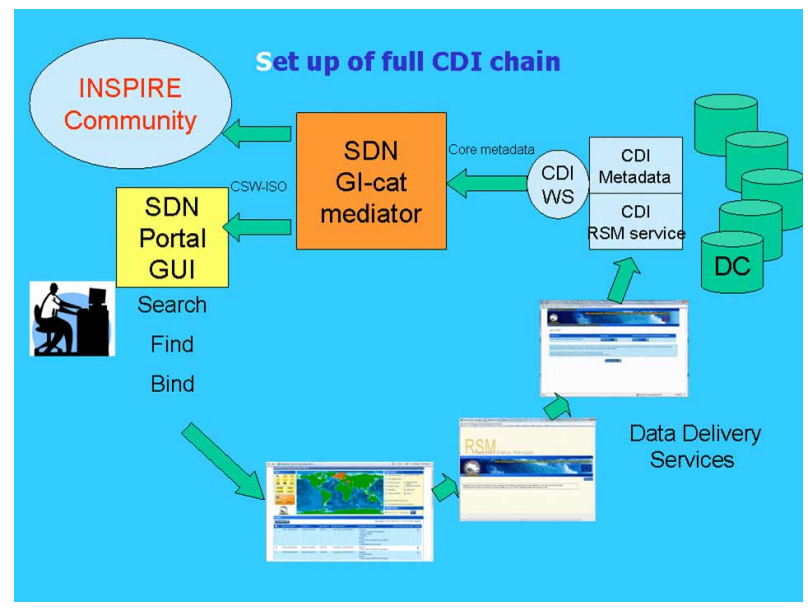

Figure 11. Application of GI-CAT in CDI V1 service chain. 


\section{SeaDataNet climatologies}

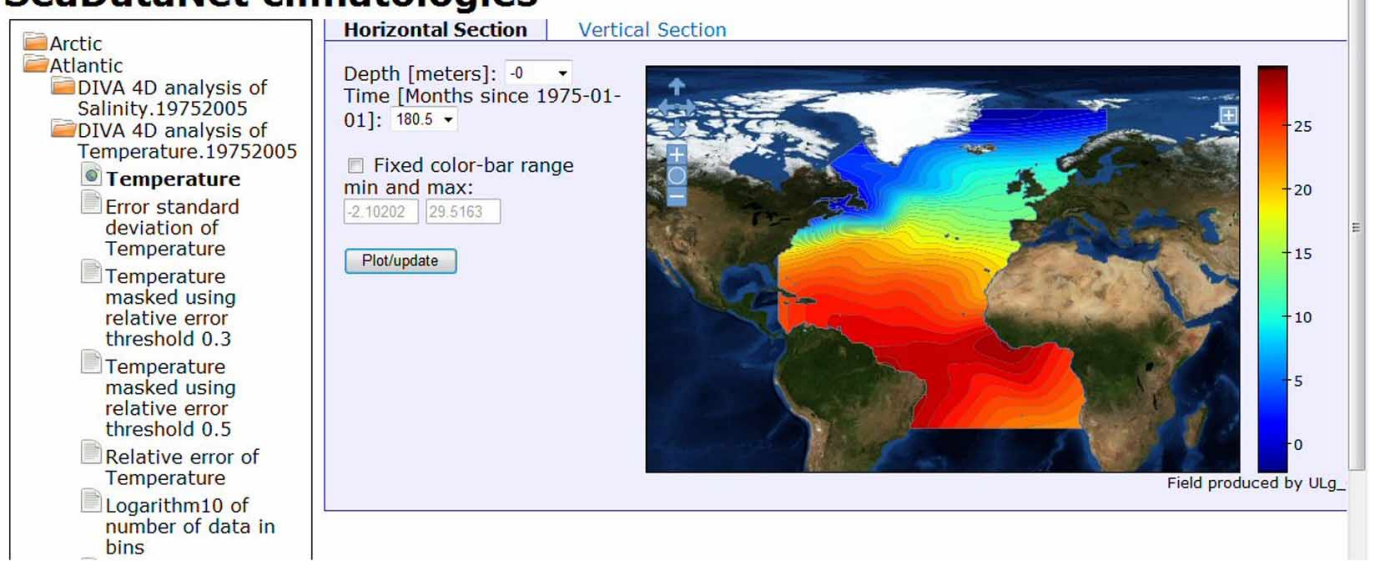

Figure 12. SeaDataNet Product examples: temperature and salinity climatologies for the Arctic Seas, Atlantic Ocean, Baltic Sea, Mediterranean Sea and the Black Sea regions. 
available data streams for complete maritime basins. The data pilots cover geology, chemistry, biology and hydrography. The SeaDataNet consortium successfully applied for implementing the EMODNET Chemical portal using the SeaDataNet network of national data centres and its new V1 infrastructure, thereby demonstrating its abilities for handling and giving users access to the requested chemical data sets and products in combination with other multidisciplinary data sets. In the coming year the first release of the chemical pilot will be prepared. The Regional Conventions (OSPAR HELCOM, Black Sea Commission) have agreed to contribute to this process.

SeaDataNet is also involved in the implementation of the other three pilots (biology, geology and hydrography), whereby common standards of SeaDataNet, for example, vocabularies and metadata will be adopted for ensuring harmonisation between the four pilots. Moreover, the SeaDataNet CDI portal will be populated by all pilots for indexing their background data sets.

SeaDataNet V1 standards and tools are also being adopted by new EU-funded projects, that will have a dedicated portal, but will also be fully integrated in the SeaDataNet infrastructure. These include:

- Up-Grade Black Sea SCENE project (2009-2011) involving six NODCs and 35 other data-holding institutes from the six Black Sea countries, to provide metadata and data access and to strengthen their national NODC networks.

- CASPINFO project (2009-2011) involving 12 institutes and private industry from the Caspian Sea region, to provide metadata and data access and to build their capacity for data management and user services.

- Geo-Seas project (2009-2012) involving 24 geological and geophysical data centres from 16 European countries (EuroGeoSurveys), to provide metadata and data access.

- EuroFleets project (2009-2012) involving 24 research institutes and data centres from 17 European countries, to optimise the management of research vessels and to streamline the flow of data from the research cruises to the data centre infrastructure.

Several SeaDataNet NODCs are participating in each project for transfer of knowledge and expertise, to secure interoperability and to learn from new developments.

\section{Acknowledgements}

This research was done on behalf of the SeaDataNet Consortium Partners (see: http:// www.seadatanet.org).

\section{Notes on contributors}

Dick M.A. Schaap, MSc, is a Managing Director of MARIS. He graduated in Coastal Engineering at the Technical University of Delft, The Netherlands. He has extensive experience in managing national and international projects for developing and implementing marine data management systems. He started his career at the North Sea directorate of the Netherlands Public Works Department. In 1986 he became coordinator of the MARIS project for improving overview and access to marine data of the North Sea in the Netherlands. Later on MARIS became private and is engaged for more than a decade in planning and 
implementing parts of a Pan-European marine data management infrastructure, involving research institutes, governmental organisations and private industry. Present projects comprise, e.g. SIMORC, SeaDataNet, Geo-Seas, EuroFleets, Humboldt and EMODNET pilots.

Dr. Roy K. Lowry is Technical Director of the British Oceanographic Data Centre, which he joined in 1981 as a scientific programmer following a BSc (Wales) and $\mathrm{PhD}$ (London) in geological sciences. From 1988 to 2000 he pioneered then developed the concept of project data management, leading the data management for many projects, such as the North Sea Project, BOFS (UK JGOFS), LOIS and OMEX and made significant contributions to international data management of chemical and biological data whilst chairing the JGOFS Data Management Task Team. Since 2000, he has developed an interest in the semantic interoperability between oceanographic data systems and currently manages the NERC Vocabulary Server, which serves many communities such as SeaDataNet, Geo-Seas and CF. 